\title{
Constatations cliniques et prise en charge de la papillopathie diabétique : Rapport de cas
}

\author{
Eva Gao, OD \\ Ancil Mathew, OD, FAAO \\ Bhagya Segu, OD, MPH, FAAO \\ Angelina Tran, OD, FAAO \\ Michael E. DeBakey \\ VA Medical Center
}

\begin{abstract}
Résumé
La papillopathie diabétique est une manifestation oculaire rare du diabète qui est associée à une diminution rapide de l'hémoglobine Alc. La présentation typique de cette condition a tendance à être asymptomatique avec une pression intracrânienne normale. Ce rapport de cas décrit un homme de 64 ans de race blanche qui s'est présenté avec une papillopathie diabétique. Comme il s'agit d'un diagnostic d'exclusion, l'imagerie par résonance magnétique, la venographie par résonance magnétique et la ponction lombaire doivent être effectuées pour exclure d'autres causes possibles d'œdème papillaire, y compris l'ischémie, l'infection, l'inflammation, l'infiltration, l'augmentation de la pression intracrânienne en raison de lésions occupant de l'espace et l'hypertension intracrânienne idiopathique.
\end{abstract}

MOTS-CLÉS :

diabète, manifestations oculaires, papillopathie, diagnostic différentiel

\section{INTRODUCTION}

La papillopathie diabétique est une cause rare d'enflure du disque optique chez les patients atteints de diabète sucré (DM ${ }^{1,2}$. Les caractéristiques cliniques typiques sont un œdème papillaire hypermétrique avec des résultats d'épreuves diagnostiques autrement non significatifs. La pathophysiologie de la papillopathie diabétique n'est pas claire, mais la documentation semble indiquer que les changements microvasculaires diabétiques qui causent une fuite de liquide dans le nerf optique seraient un facteur contributif ${ }^{1-5}$. Cette condition a également été associée à une baisse rapide de la glycé$m_{i}^{2,4,6}$. La papillopathie diabétique est considérée comme un diagnostic d'exclusion; par conséquent, les épreuves diagnostiques visant à écarter toutes les autres causes possibles de l'œdème papillaire devraient inclure l'imagerie crânienne pour la détection des lésions occupant de l'espace, la ponction lombaire pour déterminer la pression intracrânienne (PIC)et des études sérologiques du liquide céphalorachidien (LCR) pour exclure les causes infectieuses, infiltratives et inflammatoires ${ }^{1,4,7}$.

Bien que la papillopathie diabétique soit une maladie relativement bénigne qui se résorbe d'elle-même et qui a un impact visuel minimal, les cas ont démontré que l'utilisation d'injections intravitréennes de bévacizumab peut accélérer la résolution chez les personnes ayant une perte visuelle importante ${ }^{8,9}$.

\section{RAPPORT DE CAS}

Un homme de 64 ans de race blanche s'est présenté pour un examen postopératoire d'un mois à la suite d'une chirurgie de la cataracte à son œil gauche (OS). Il a signalé n'avoir aucun changement visuel ni préoccupation et utilisait à ce moment une suspension ophtalmique à $1 \%$ d'acétate de prednisolone une fois par jour dans l'œil gauche depuis l'intervention. Les antécédents oculaires du patient faisaient état d'un DM de type II sans manifestations oculaires et d'une extraction de cataracte avec implants de lentille intraoculaire de la chambre postérieure dans les deux yeux (OU). Le patient avait des antécédents de DM de type II (depuis10 ans), d'arthrite rhumatoïde, d'hypothyroïdie et d'hypertension. Ses médicaments compre- 
naient l'amlodipine, le glipizide, la lévothyroxine, le losartan, la metformine, le méthotrexate et la saxagliptine. Il était allergique à la pénicilline et au lisinopril. Ses taux d'hémoglobine d'Alc (HbAlc) avaient diminué de 1,5\% au cours du mois de la présentation comparativement au niveau enregistré trois mois auparavant (tableau 1).

Sa meilleure acuité corrigée était de 20/20-2 à l'œil droit (OD) et de 20/30+2 à l'œil gauche. Sa meilleure acuité corrigée OS était de 20/25 avant de développer une cataracte significative et de subir une chirurgie de la cataracte. Les pupilles étaient de diamètre égal et réactives à la lumière et qu'elles ne présentaient aucun défaut pupillaire afférent. Les mouvements extraoculaires et les champs visuels de confrontation étaient pleins. Les pressions intraoculaires par tonométrie d'applanation de Goldmann étaient de $17 \mathrm{mmHg}$ OD et $15 \mathrm{mmHg}$ OS. Le segment antérieur des yeux n'avait rien d'anormal et il était pseudophakique dans les deux yeux.

L'examen du fond de l'œil du nerf optique a révélé un œè̀me papillaire OD de grade 4 avec hémorragie péripapillaire de $360^{\circ}$ et un œdème papillaire OS de grade 2 avec quelques hémorragies (figures 1.2), qui ont été classés à l'aide de l'échelle de Frisen modifiée (tableau 3). Le pôle postérieur de la rétine présentait de multiples hémorragies dispersées et une tortuosité des vaisseaux OU. Une tomographie par cohérence optique en domaine spectral (TCO-DS) de la couche de fibres nerveuses rétiniennes (CFNR) a montré un épaississement important de la CFNR, avec une valeur globale de $244 \mu \mathrm{m}$ OD et de $210 \mu \mathrm{m}$ OS. La TCO-DS de la macula était normale sans œè̀me maculaire OU.

Le patient a reçu un diagnostic d'œdème papillaire bilatéral et de rétinopathie diabétique non proliférante modérée (RDNP) sans œdème maculaire, qui était plus grave dans l'œil droit, selon l'Étude sur le traitement précoce de la rétinopathie diabétique (ETDRS). En raison de ces constatations, un examen plus approfondi des systèmes a été effectué. Il a affirmé n'avoir aucun mal de tête, nausée, douleur oculaire, malaise ou perte de vision temporaire. Aucun supplément de vitamine A, aucun corticostéroïde ou usage récent de tétracycline n’a été signalé. Sa tension artérielle était de 126/66 mmHg au moment de la consultation. Il a par la suite été admis à l'urgence pour un examen neurologique, y compris une imagerie, une ponction lombaire (PL) et une numération sanguine complète (NSC), afin de déterminer la cause de l'œè̀me papillaire.

\section{Épreuves diagnostiques}

La tomodensitométrie (TDM), l'imagerie par résonance magnétique (IRM) et la venographie par résonance magnétique (VRM) de la tête n'ont révélé aucune anomalie qui aurait pu expliquer l'enflure du nerf optique. Son examen neurologique n'a rien révélé non plus. La ponction lombaire (PL) a révélé une pression d'ouverture de $16 \mathrm{~cm}^{\mathrm{de}} \mathrm{H}_{2} \mathrm{O}$ (plage normale chez les adultes de 6 à $25 \mathrm{~cm}$ de $\mathrm{H}_{2} \mathrm{O}$ ) et le liquide céphalorachidien (LCR) n'a rien révélé. Les résultats de la NSC étaient normaux. Les résultats de ces tests ont écarté la possibilité que ses symptômes soient dus à des lésions, des infections ou une augmentation de la pression intracrânienne due à des lésions occupants de l'espace.

\section{Consultations de suivi}

Lors de ses visites de suivi, sa MAVC est demeurée stable. L'œdème du nerf optique a diminué à chaque visite subséquente à 1 mois et à 3 mois, et s'était résorbé au cours du suivi de 5 mois. La résolution de l'œdème papillaire est demeurée stable à son suivi de 7 mois. Le test de champ visuel de Humphrey 24-2 SITA (Swedish Interactive Testing Algorithm) FASTER, réalisé lors de son suivi de 7 mois, a montré des déficit épars dans la région supérieure OD et des déficit épars à la bordure OS avec une bonne fiabilité; toutefois, il s'agissait du premier test du champ visuel du patient et un test répété sera bénéfique. Les figures 1-13 et les tableaux 2 et 4 montrent l'amélioration continue de l'œdème papillaire au cours des sept mois suivant la présentation initiale.

Après des épreuves approfondies visant à exclure toute autre étiologie possible, le diagnostic final était la papillopathie diabétique. Cela correspond à l'absence de symptômes chez le patient, à la RDNP modérée et à la diminution rapide de ses niveaux de HbAlc. Au moment de son suivi de cinq mois, l'œdème papillaire s'était résorbé et était demeuré stable à son suivi de sept mois. 
Tableau 1 : Concentrations de HbAlc au cours de la dernière année.

\begin{tabular}{|c|c|}
\hline Date & HbAlc (\%) \\
\hline Avr. 2018 & 7,8 \\
\hline Nov 2018 & 7,8 \\
\hline Juin 2018 & 9,3 \\
\hline Sept 2018* & 7,8 \\
\hline Jan 2019 & 9,4 \\
\hline
\end{tabular}

*Dernier HbAlc avant la présentation
Tableau 2 : Épaisseur globale de la CFNR au fil du temps

\begin{tabular}{|l|c|c|}
\hline & Eil droit $(\mu \mathrm{m})$ & Eil gauche $(\mu \mathrm{m})$ \\
\hline Visite initiale & 244 & 210 \\
\hline Suivi d'un mois & 165 & 131 \\
\hline Suivi de 3 mois & 118 & 112 \\
\hline Suivi de 5 mois & 98 & 101 \\
\hline Suivi de 7 mois & 101 & 98 \\
\hline
\end{tabular}

Tableau 3 : Échelle de Frisen modifiée

\begin{tabular}{|l|l|}
\hline Échelle de Frisen modifiée \\
\hline Grade & Caractéristiques \\
\hline 0 - Normal & CFN radiale sans tortuosité \\
\hline - Minime & $\begin{array}{l}\text { Pas d'élévation des bordures } \\
\text { Obscurcissement de la bordure nasale du disque } \\
\text { Perturbation de la disposition normale de la CFN radiale } \\
\text { Marge de disque temporelle normale }\end{array}$ \\
\hline 2- Faible & $\begin{array}{l}\text { Élévation de la bordure nasale } \\
\text { Obscurcissement de toutes les bordures } \\
\text { Halo péripapillaire } \\
\text { Aucun obscurcissement majeur des vaisseaux }\end{array}$ \\
\hline $3-$ Modéré & $\begin{array}{l}\text { Élévation de toutes les bordures } \\
\text { Obscurcissement de toutes les bordures } \\
\text { Halo péripapillaire } \\
\text { Obscurcissement d'un ou de plusieurs segments d'un vaisseau sanguin majeur à la sortie du disque }\end{array}$ \\
\hline $4-$ Marqué & $\begin{array}{l}\text { Élévation de toute la tête du nerf optique } \\
\text { Obscurcissement de toutes les bordures } \\
\text { Halo péripapillaire } \\
\text { Obscurcissement total d'un vaisseau sanguin majeur sur le disque }\end{array}$ \\
\hline 5 - Grave & $\begin{array}{l}\text { Obscurcissement total d'un vaisseau sanguin majeur sur le disque et obscurcissement partiel de tous } \\
\text { les vaisseaux sur le disque }\end{array}$ \\
\hline
\end{tabular}

Tableau 4 : Grade de l'œdème papillaire au fil du temps basé sur l'échelle de Frisen modifiée

\begin{tabular}{|l|c|c|}
\hline & Eil droit & Eil gauche \\
\hline Visite initiale & 4 & 2 \\
\hline Suivi de 1 mois & 2 & 1 \\
\hline Suivi de 3 mois & 1 & 1 \\
\hline Suivi de 5 mois & 0 & 0 \\
\hline Suivi de 7 mois & 0 & 0 \\
\hline
\end{tabular}


Figures 1 à 8 : Photographies du fond de l'œil

Figure 1

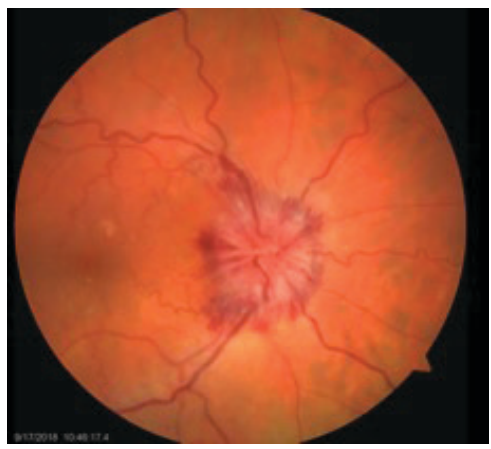

Figure 4

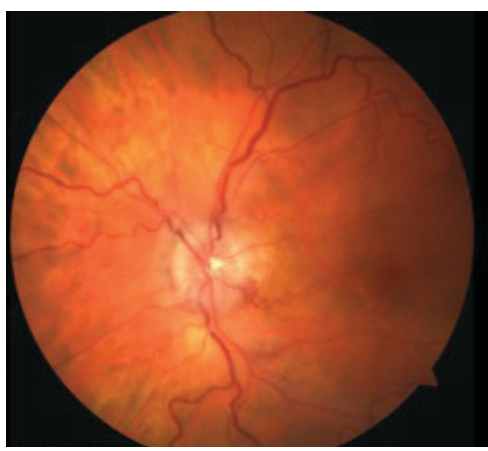

Figure 7

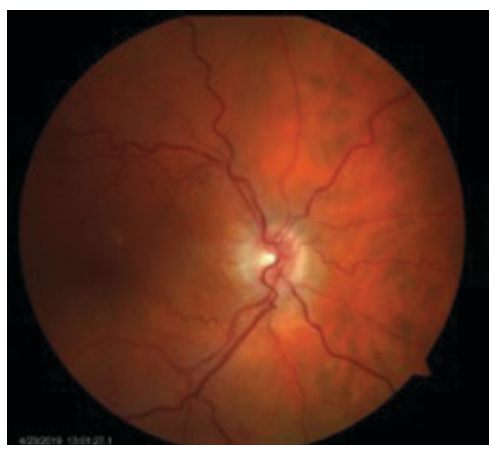

Figure 2

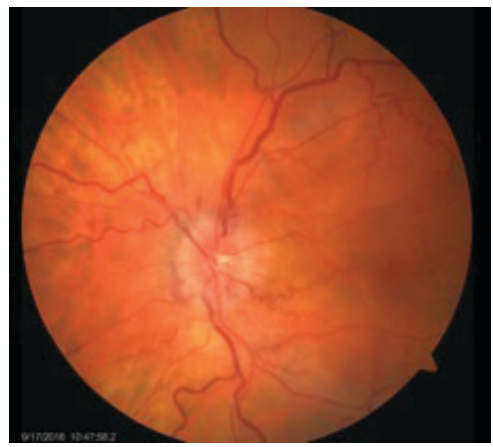

Figure 5

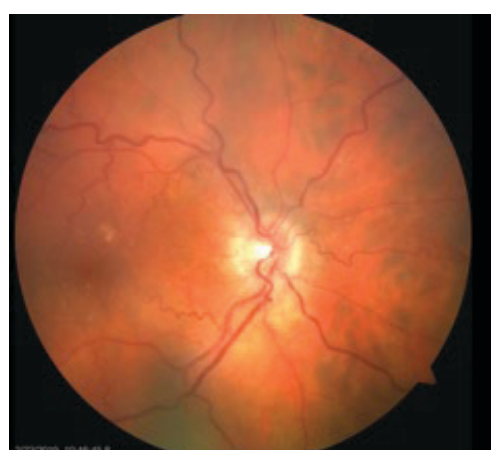

Figure 8

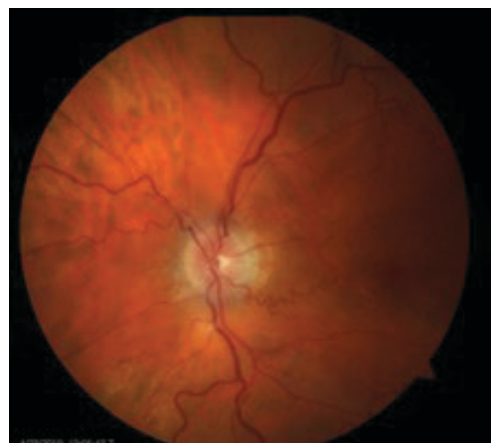

Figure 3

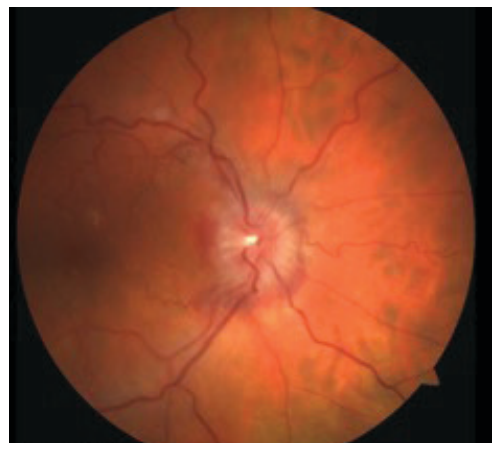

Figure 6

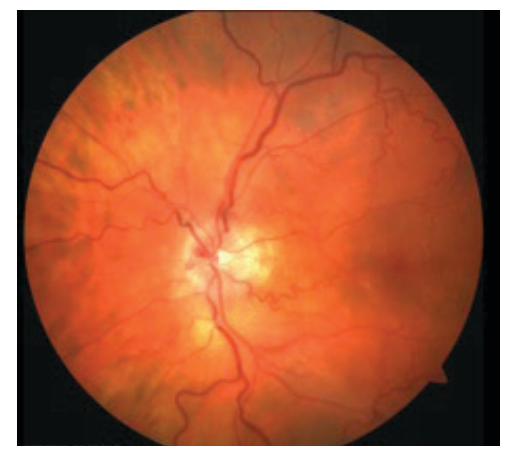


Figure 9 : TCO DS Spectralis à la première visite, œil droit

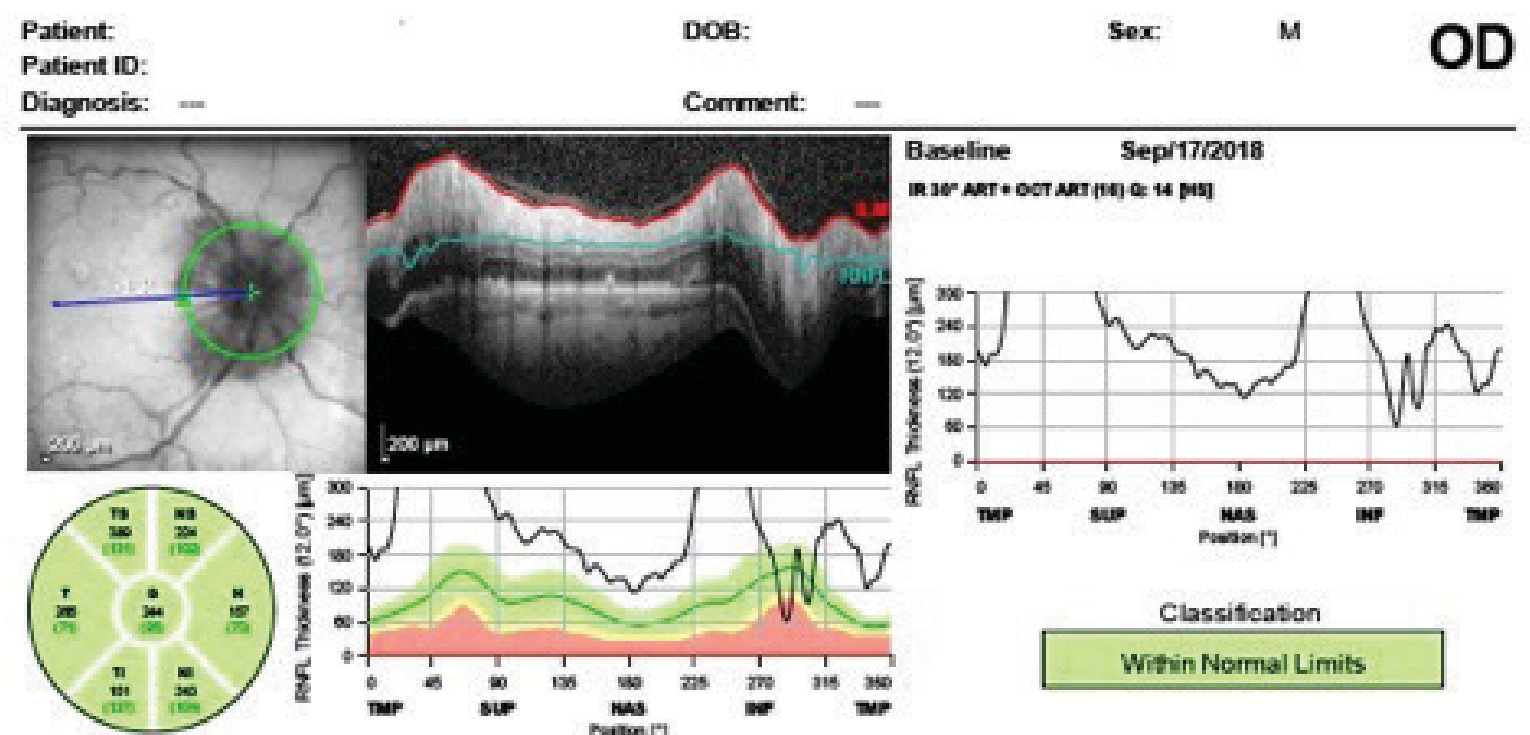

Figure 10 : TCO DS Spectralis à la première visite, oeil gauche

Patient:

\section{Patient ID:}

Diagnosis: - -
DOB:

Comment: -

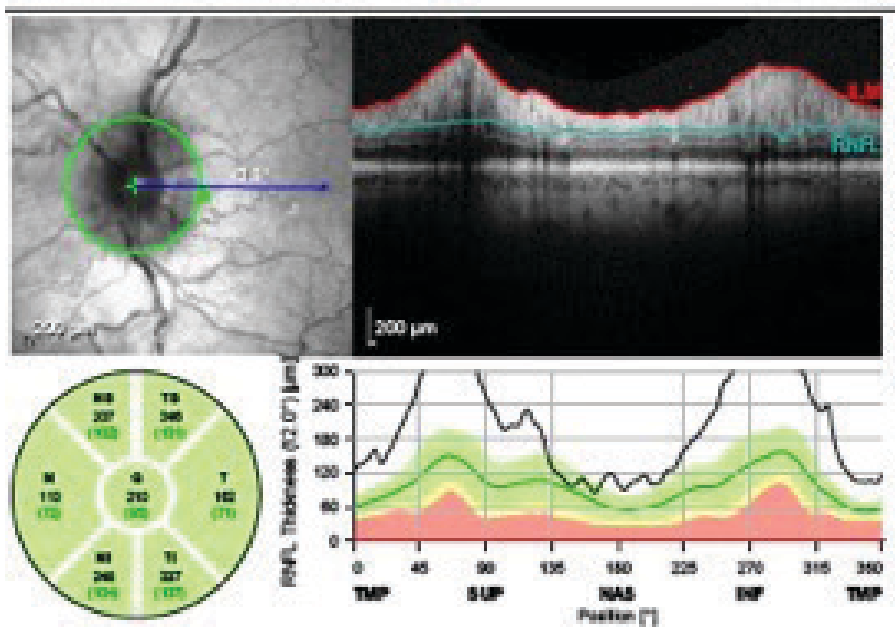

Sex:

M

Sep/17/2018

Baseline seprot oct ant

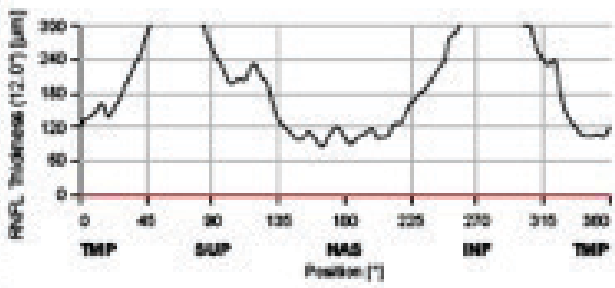

Classification

Within Normal Limits 
Figure 11: TCO DS Spectralis à la visite de suivi de 7 mois

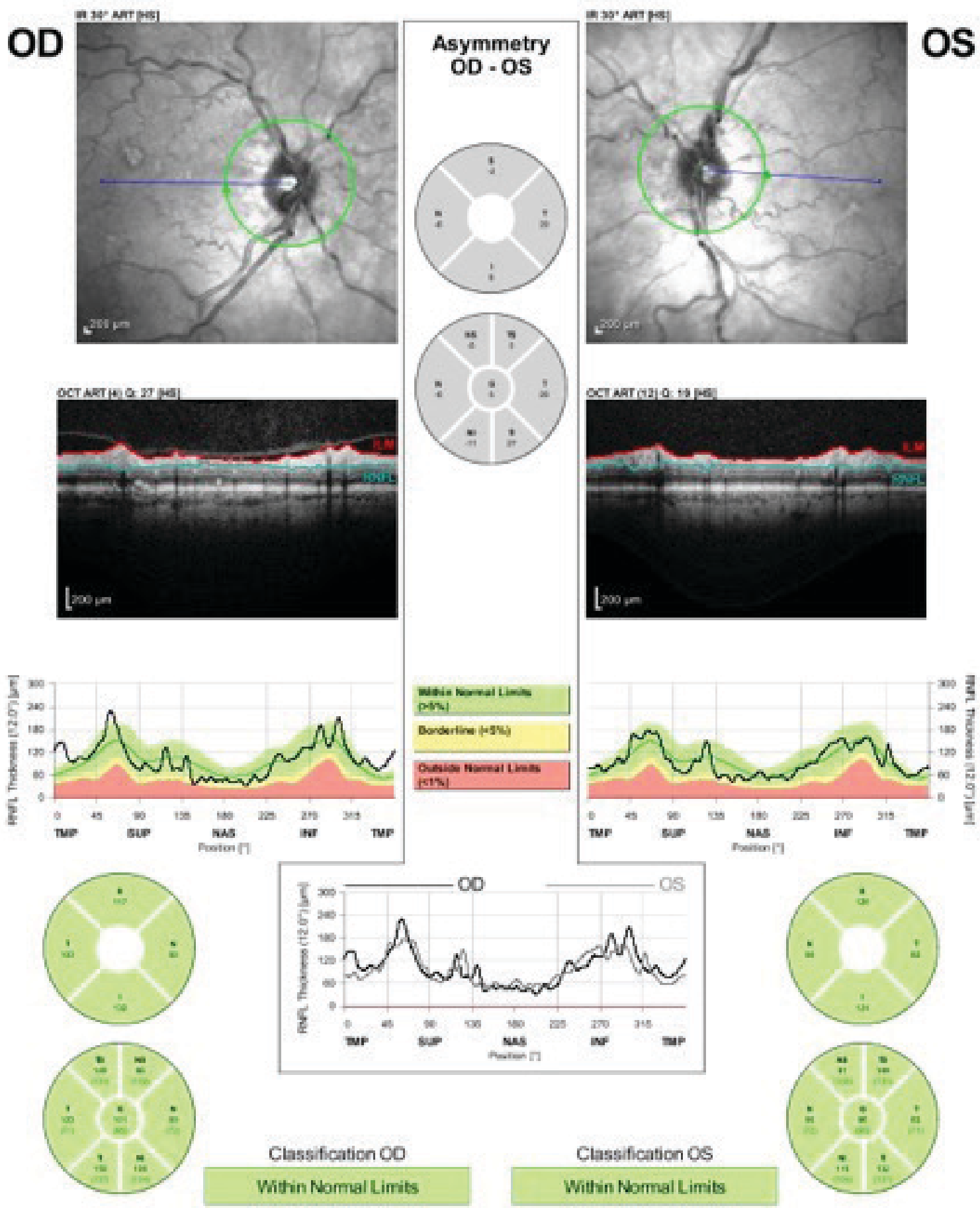



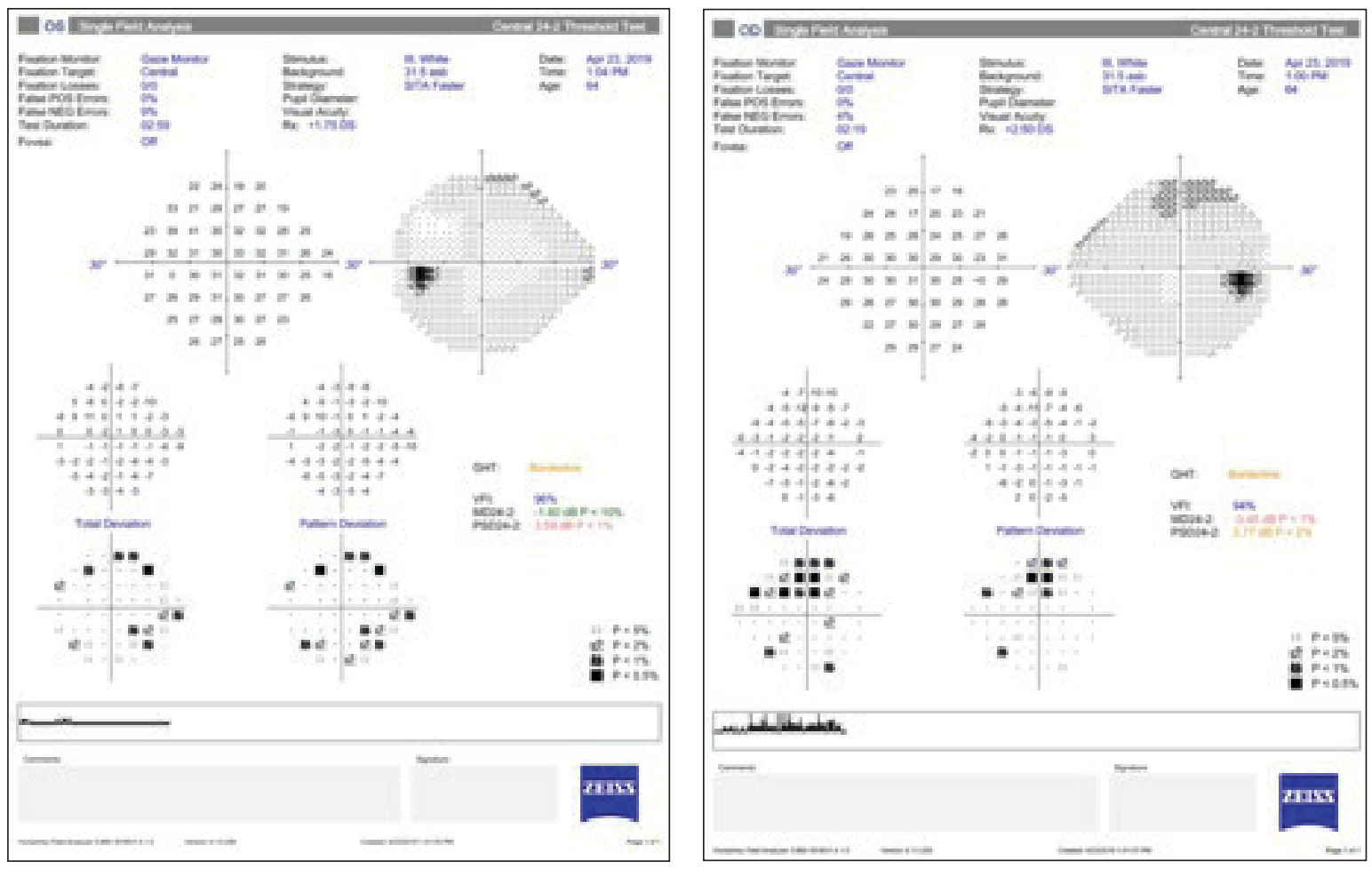

DISCUSSION

La papillopathie diabétique est une cause rare d'œè̀me papillaire que l'on observe chez 1,4 \% des patients diabétiques; elle est considérée comme un diagnostic d'exclusion ${ }^{1,2,10}$. La maladie peut affecter les patients atteints de diabète de type I ou II sans aucune prédilection en matière d'âge, de race ou de sexe et est souvent sous-diagnostiquée en raison de sa nature essentiellement asymptomatique et transitoire ${ }^{1,2}$. Les patients atteints de cette affection ne présentent généralement que des troubles visuels ou une perte de champ visuel minimes, voire aucun, et on n’observe généralement aucun défaut pupillaire afférent ${ }^{3}$. Les acuités visuelles peuvent être touchées dans les cas d'œdème maculaire concomitant, et une tache aveugle agrandie est un défaut typique du champ visuel.

La papillopathie diabétique peut se présenter unilatéralement ou bilatéralement, et elle est habituellement accompagnée de signes de RDNP bénigne (bien qu'elle puisse apparaître avec n'importe quel niveau de rétinopathie ou en l'absence de rétinopathie) ${ }^{1}$. Les résultats caractéristiques de l'examen du fond de l'œil comprennent l'enflure hyperémique du disque optique et des vaisseaux télangiectatiques sur le disque ou autour de celui-ci ${ }^{2,3}$. Un œedème maculaire cliniquement significatif est associé à 50 à $70 \%$ des yeux atteints de papillopathie diabétique ${ }^{1,2}$. L'angiographie par fluorescéine du fond de l'œil (AF) montre une hyperfluorescence précoce focale ou diffuse sur le disque avec une fuite tardive des vaisseaux télangiectatiques ${ }^{2}$.

La pathogenèse de la papillopathie diabétique demeure incertaine, mais elle est probablement due à des changements microvasculaires diabétiques. Une revue de la documentation révèle que l'œdème papillaire est causé par une perturbation vasculaire rétinienne superficielle accompagnée d'une fuite transitoire de liquide dans et autour de la tête du nerf optique ${ }^{1}$. Certaines études semblent indiquer que l'œè̀me du disque a une origine plus profonde dans la tête du nerf optique, en raison d'une certaine atteinte vasculaire qui perturbe le flux axoplasmique ${ }^{1}$. Bien que le premier rapport sur la papillopathie diabétique remonte à 1971, on se demande encore s'il s'agit d'une entité propre, d'une manifestation de la rétinopathie diabétique ou d'une forme de neuropathie optique ischémique antérieure (NOIA). Le contrôle métabolique peut aussi contribuer au développement de la papillopathie diabétique, car on a découvert qu'il y a une association marquée entre la papillopathie diabétique et les patients présentant une réduction drastique récente de la $\mathrm{HbAlc}$ et des rapports cupule-disque faibles ${ }^{2,4,6,11}$. Par conséquent, une réduction 
de la HbAlc d'au plus 1,0 \% par trimestre est recommandée ${ }^{4}$. Dans ce cas, les niveaux de HbAlc du patient avaient diminué de $1,5 \%$ au cours du mois où il s'est présenté par rapport au niveau enregistré 3 mois auparavant.

\section{Notation de l'œè̀me papillaire}

L'œdème papillaire peut être évalué qualitativement à l'aide de l'échelle de Frisen modifiée (MFS) ou quantitativement au moyen de la $\mathrm{TCO}^{12}$. La MFS utilise les caractéristiques clés pour attribuer une note de 0 (disque optique normal) à 5 (œdème grave), comme l'indique le tableau $3^{12}$. Les photographies du fond de l'œil sont utiles lorsque l'on utilise la MFS. La MFS convient mieux à la catégorisation de l'œedème de grade plus élevé et à l'observation des principales caractéristiques, tandis que la TCO est plus efficace pour l'œè̀me de grade plus bas, quand les changements subtils du nerf optique sont plus difficiles à discerner ${ }^{12}$. De plus, l'algorithme de la TCO pour le profil d'épaisseur de la CFNR donne souvent de mauvais résultats avec les œedèmes de haut grade ${ }^{12}$. L'épaisseur moyenne de la CFNR sur le TCO-DS Spectralis dans la population moyenne est de $97,3 \pm 9,6 \mu \mathrm{m}^{13}$. Lors de sa présentation initiale, le patient avait un œdème de grade 4 et un œdème de grade 2 sur la MFS, et l'épaisseur globale de la CFNR était de $244 \mu \mathrm{m}$ pour l'œil droit et de $210 \mu \mathrm{m}$ pour l'œil gauche. Au départ, la MFS convenait mieux à la surveillance en raison d'une possible défaillance de l'algorithme de la TCO avec un œdème de disque de grade plus élevé.

L’utilisation combinée de la MFS, des photographies du fond de l'œil et de la TCO fournit la meilleure documentation pour évaluer l'évolution clinique globale de l'œè̀me papillaire.

\section{Diagnostic différentiel}

Comme la papillopathie diabétique est un diagnostic d'exclusion, il est essentiel d'exclure d'autres causes possibles comme la NOIA, la névrite optique et l'augmentation de la pression intracrânienne causée par les lésions occupant de l'espace, l'infection et l'inflammation ${ }^{4}$. Les patients qui ont une NOIA présentent généralement une perte de vision importante et soudaine avec un défaut du champ visuel altitudinal dense ${ }^{2}$.La neurite optique est habituellement associée à la douleur oculaire, à la dyschromatopsie et à la perte du champ visuel'1. L'imagerie crânienne (IRM, TDM par VRM), l'analyse du LCR et les analyses sanguines peuvent exclure une augmentation de la pression intracrânienne causée par une lésion occupant de l'espace, une infection ou l'inflammation. L'hypertension intracrânienne idiopathique présente une pression d'ouverture accrue sur le PA et est souvent associée à des maux de tête, à la diplopie et au malaise'. L'IRM et la TDM peuvent aider à détecter les lésions occupant de l'espace et la VEM peut aider à détecter la thrombose des sinus veineux ${ }^{14}$. Il existe une grande variété de causes infectieuses comme la sarcoïdose, la trichinose et la toxoplasmose ${ }^{15}$. Une étiologie infectieuse peut être confirmée par des épreuves de laboratoire comme une NSC, le taux de sédimentation des érythrocytes, le dosage de la protéine C-réactive (CRP), la réaction en chaîne de la polymérase (PCR) et des tests sérologiques ${ }^{16}$. L'inflammation, comme dans le cas de la méningite, peut augmenter la PIC en réduisant la réabsorption du LCR ${ }^{15}$. L'œdème pseudo-papillaire est l'élévation anormale du disque optique dû aux drusen et peut être diagnostiqué par un B-scan ou une autofluorescence du fond d'œil (FAF) du nerf optique ${ }^{17}$. Certains médicaments comme les œstrogènes, les tétracyclines, les corticostéroïdes et la vitamine A peuvent aussi causer un œdème papillaire ${ }^{18}$.

\section{Traitement}

La papillopathie diabétique est souvent surveillée sans intervention en raison de sa nature autolimitative et bénigne $^{8}$. Le temps de résolution peut varier de 3 à 12 mois après la présentation initiale ${ }^{3}$. Les patients peuvent avoir une pâleur légère des disques optiques à la résolution en cas d'œedème papillaire prolongé ${ }^{19}$. Aucune corrélation entre le grade de l'œdème et l'acuité initiale ou le résultat visuel n'a été établie ${ }^{19}$.

Dans les cas de perte de vision, les injections d'un facteur de croissance endothélial antivasculaire (anti-VEGF) peuvent être bénéfiques ${ }^{8,9}$. Les yeux dans lesquels on a injecté du bévacizumab intravitréen ont montré une régression marquée de l'œdème du disque et une amélioration de l'acuité visuelle au suivi de 2 semaines ${ }^{8,9}$. Toutefois, d'autres études seront nécessaires pour prouver son efficacité et son innocuité dans la papillopathie diabétique.

\section{CONCLUSION}

Le présent rapport de cas traite du diagnostic et de la prise en charge d'un patient présentant des résultats caractéristiques de la papillopathie diabétique bilatérale, c.-à-d. un œedème papillaire hyperémique sans constatations particulières quant aux résultats des tests neurologiques, à l'imagerie crânienne, à la pression intracrânienne, la sérologie et la composition du liquide céphalorachidien. La prévalence mondiale du diabète a augmenté rapidement au cours des dernières décennies, et il est impératif que les fournisseurs de soins oculovisuels connaissent certaines des complications oculaires les moins courantes du diabète, y compris la papillopathie diabétique. De plus, il est 
essentiel de différencier la papillopathie diabétique, une maladie bénigne qui guérit d'elle-même, des maladies qui mettent la vie en danger et qui peuvent causer un odème papillaire.

DIVULGATIONS FINANCIÈRES :

L'auteur ou les auteurs n'ont aucun intérêt commercial ou exclusif à l'égard des sujets abordés dans le présent article.

\section{RÉFÉRENCES}

1. Regillo CD, Brown GC, Savino PJ, et al. Diabetic Papillopathy. Arch Ophthalmol 1995;113:889-895.

2. Bayraktar Z, Alacali N, Bayraktar S. Diabetic Papillopathy in Type II Diabetic Patients. Retina 2002;22:752-758.

3. Slagle WS, Musick AN, Ecjermann DR. Diabetic Papillopathy and Its Relation to Optic Nerve Ischemia. Optom Vis Sci 2009;86:E395E403.

4. Ostri C, Lund-Anderson H, Sander B, et al. Bilateral Diabetic Papillopathy and Metabolic Control. Ophthalmology 2010;117:2214-2217.

5. Lubow M, Makley TA. Pseudopapilledema of Juvenile Diabetes Mellitus. Arch Ophthalmol 1971;85:417-422.

6. Mallika PS, Aziz S, Asok T, et al. Severe Diabetic Papillopathy Mimicking Non-Arteritic Anterior Ischemic Optic Neuropathy (NAION) in a Young Patient. Med J Malaysia 2012;67:228-230.

7. Portelli M, Papageorgiou PN. An Update on Idiopathic Intracranial Hypertension. Acta Neurochir 2017;159:491-499.

8. Yildirim M, Kilic D, Dursun ME, et al. Diabetic Papillopathy Treated with Intravitreal Ranibizumab. Int Med Case Rep J 2017;10:99-103.

9. Al-Dhibi, H, Khan AO. Response of Diabetic Papillopathy to Intravitreal Bevacizumab. Middle East African J Ophthalmol 2011;18:243-245.

10. Al-Hinqi AS, Al-Abri MS, Al-Hajri RH. Diabetic Papillopathy with macular edema treated with intravitreal bevacizumab. Oman J Ophthalmol 2011;4(3):135-138.
11. Funatsu H, Yamashita H, Ohashi Y, Ishigaki T. Effect of rapid glycemic control on progression of diabetic retinopathy. Jpn J Opthalmol 1992;36:356-367.

12. Scott CJ, Kardon RH, Lee AG, et al. Diagnosis and Grading of Papilledema in Patients with Raised Intracranial Pressure Using Optical Coherence Tomography vs Clinical Expert Assessment Using a Clinical Staging Scale. Arch Ophthalmol 2010;128(6):705-711.

13. Alasil T, Wang K, Keane PA, et al. Analysis of Normal Retinal Nerve Fiber Layer Thickness by Age, Sex, and Race Using Spectral Domain Optical Coherence Tomography. J Glaucoma 2013;22:532-541.

14. Higgins JNP, Gillard JH, Owler BK, et al. MR venography in idiopathic intracranial hypertension: unappreciated and misunderstood. J Neurol Neurosurg Psychiatry 2004;75:621-625.

15. Rigi M, Almarzouqi SJ, Morgan ML, et al. Papilledema: epidemiology, etiology, and clinical management. Eye Brain 2015;7:47-57.

16. Kahloun R, Abroug N, Ksiaa I, et al. Infectious optic neuropathies: a clinical update. Eye Brain 2015;7:59-81

17. Robolleda G, Kawasaki A, Juan V, et al. Optical Coherence Tomography to Differentiate Papilledema from Pseudopapilledema. Curr Neurol Neurosci Rep 2017;17(74):1-13.

18. Thon OR, Gittinger JW. Medication-Related Pseudotumor Cerebri Syndrome. Semin Ophthalmol 2017;31(1):134-143.

19. Barr CC, Glaser JS, Blankenship G. Acute Disc Swelling in Juvenile Diabetes. Arch Ophthalmol 1980;98:2185-2192. 